\title{
The Combined Effect of Buoyancy and Excess Pore Pressure in Facilitating Soil Liquefaction
}

\author{
Ben Zeev S. ${ }^{1}$, Goren L. ${ }^{2}$, Parez S. ${ }^{3}$, Toussaint R. ${ }^{4}$, Clément C. ${ }^{4}$ and \\ Aharonov E. 1 \\ ${ }^{1}$ Institute of Earth Science, Hebrew University of Jerusalem, Israel; \\ e-mail: shahar.benzeev@mail.huji.ac.il; \\ ${ }^{2}$ Geological \& Environmental Science, Ben-Gurion University of the Negev, Israel; \\ ${ }^{3}$ University of Chemistry and Technology Prague, The Czech Republic; \\ ${ }^{4}$ Université de Strasbourg, CNRS, Institut de Physique du Globe de Strasbourg, \\ UMR7516, F-67000 Strasbourg, France;
}

\begin{abstract}
Soil liquefaction is a devastating earthquake hazard, commonly causing tilting, sinking and floating of infrastructure. The classical mechanism for liquefaction requires undrained and loosely packed soil, that upon shear experiences elevated, lithostatic, pore pressure and consequently zero effective stress. However, some field and experimental observations cannot be explained by this mechanism. These include liquefaction of precompacted soils, liquefaction under drained conditions, repeated liquefaction events, and liquefaction triggered by small seismic energy density. A recent study suggests a new mechanism for soil liquefaction that arises only from buoyancy effects of fluids plus grain accelerations, where the term "liquefaction", used as its phenomenological field definition, refers to a macroscopic transition from rigid to fluid-like behavior. We extend that study and seek a unifying mechanism for field observed liquefaction that accounts both for the buoyancy effect and for elevated pore pressure, though not necessarily with lithostatic values. To achieve this goal, we use a coupled fluid flow and granular dynamics numerical model to study the effect of pore pressure on the sinking of a large object ("intruder") into a drained densely packed granular system, undergoing cyclic shearing. Results show that despite the drained conditions pore pressure rises during shaking. Although pore pressure remains well below lithostatic values, the soil liquefies, as identified macroscopically by intruder sinking to its isostatic position. Even simulations with buoyancy effects alone show liquefaction and intruder sinking under certain conditions, yet inclusion of pore-pressure effects add to the buoyancy effect, and is seen to enhance liquefaction and promote intruder sinking.
\end{abstract}




\section{INTRODUCTION}

Soil liquefaction is a natural hazard that accompanies many earthquakes, with potentially destructive consequences that include tilting, sinking and uplifting of infrastructure. Two different definitions are commonly used for liquefaction: The first is a phenomenological definition, used practically in the field. It identifies liquefaction via observations of macro-scale changes in the rheological response of soils, from rigid to a fluid-like slurry during or following an earthquake. In contrast, the second definition is mechanistic in nature. It is used in laboratory tests and relates liquefaction to rheological change caused by pore pressure rise to lithostatic levels (Youd \& Idriss 2001, Martin et al. 1975). Clément et al. 2017 a,b suggested that the two definitions do not always coincide, and that many of the field-observed liquefaction events may occur at relatively low pore pressure. Indeed, the pore pressure (PP) mechanism for liquefaction requires soils that are initially loose and effectively undrained (Youd \& Idriss 2001). The loose soil skeleton compacts during shaking, decreasing pore volume and increasing PP. The undrained conditions prevent fluid escape from the compacting pore volume, allowing PP to reach lithostatic values. Yet, this PP mechanism fails to explain many field and experimental observations of liquefaction: (1) Liquefaction in pre-compacted soils (Soga 1998), (2) Under drained conditions, see e.g. demo at https://www.youtube.com/watch? $\mathrm{v}=\mathrm{cONq} 231 \mathrm{dn} 6 \mathrm{w}$, (3) Repeated liquefaction events (Obermeier 1996), and (4) Far-field liquefaction that occurs despite small seismic energy input (Wang 2007).

Thus Clément et al. 2017a,b, suggested an alternative liquefaction mechanism, that may explain those previously unexplained occurrences of liquefaction. This new mechanism for liquefaction requires fluid, but does not require PP increase. To test the new mechanism, Clément et al. (2017a,b) probed the conditions for the onset of liquefaction using the macroscale sinking pattern of an "intruder" (a big grain), placed on the top of a saturated layer composed of smaller grains, to horizontal cyclic shear. This was done using theoretical analysis, experiments and numerical simulations. The simulations were based on the Discrete Element Method algorithm (DEM) (Cundall \& Strack 1979), modified to include the buoyancy force of the fluid as it acts on the grains and the intruder, proportional to their immersed volume in the fluid. The results of that work show an alternative mechanism for liquefaction that arises from grain acceleration and buoyancy forces alone, as PP (deviating from hydrostatic) was not included in the Clément et al. theory and simulations, but liquefaction in the phenomenological sense, was still observed. Thus, it appears that PP rise is not a necessary condition for liquefaction. However, as many field observations show evidence for elevated PP during 
liquefaction [e.g. Obermeier 1996, Holzer et al. 1989], we hypothesize that PP rise can enhance liquefaction and expand the dynamic regime over which buoyancy-acceleration triggered liquefaction occurs. It is therefore suggested that the new buoyancyacceleration mechanism and the more classical elevated PP mechanism combine to span a spectrum of conditions that can lead to soil liquefaction.

In the current work, we explore this idea by extending the numerical work of Clément et al. to include beyond hydrostatic pore pressure effects. We present preliminary results from the extended buoyancy - pore pressure model, showing the capacity of elevated pore pressure, even when significantly lower than the lithostatic stress, to enhance the sinking of an intruder.

\section{RESULTS FROM CLÉMENT ET AL. 2017}

Clément et al. 2017a,b simulated the response of a densely packed saturated granular media to earthquake shaking using a modified DEM, accounting only for the buoyancy effect of the fluid but not including PP. In their simulations, they identified the onset of soil liquefaction both via micromechanics and via the sinking of an intruder lying on top of a saturated granular layer, which undergoes horizontal cyclic shaking. Liquefaction in the simulations was defined by following the intruder sinking pattern, in a similar way to phenomenological field observations of liquefaction during and following earthquakes. Their simulation results show that the dynamic response of the grains and the intruder depends on the horizontal acceleration. For low acceleration, the grains and the intruder move together with almost no sliding along granular contacts. As a result, the intruder doesn't sink at all. At higher horizontal acceleration, high relative velocity between the grains is observed, with the exception of the region surrounding the intruder, where the grains move semi synchronously with the intruder. The outcome of this dynamics is that the intruder sinks towards its isostatic position. Intruder sinking is facilitated by grain-grain contact sliding that allows rearrangement of the medium surrounding the intruder. With the buoyancy effect, granular sliding is easier, as the normal contact force between grains is reduced with respect to a dry layer. Less sliding occurs in the vicinity of the intruder because the intruder is only partially immersed, and the buoyancy force acting to reduce the normal contact forces between the intruder and its neighboring grains is smaller. Since these simulations don't include PP, their results demonstrate that liquefaction, with sinking structures, can occur without elevated PP rise beyond hydrostatic values. This buoyancy dependent liquefaction mechanism may explain liquefaction of pre-compacted soil, under drained conditions and repeated liquefaction events. It further predicts liquefaction with low energy and low earthquake- 
induced peak ground accelerations, that can possibly explain far field liquefaction events that occurred despite small seismic energy input (Wang 2007).

\section{INTRUDER AND PORE PRESSURE SIMULATION METHOD}

Solid Phase. In our new simulations we use a similar DEM for the grains as in Clément et al. 2017a,b, but add to it the pore pressure effect. The grains are modeled as spheres with a linear elastic contact model. A velocity dependent damping is added to the normal contact force, and a threshold friction law is added to the tangential force that allows sliding when the shear force surpasses a frictional criterion. Grain motion is determined by time integration of the linear (eq. 1) and rotational (eq. 2) momentum conservation equations.

$m_{i} \dot{u}_{i}=m_{i} g-V_{i m m} \rho_{f} g+\sum_{j} F_{i j}-\frac{\nabla P \cdot V_{i}}{1-\phi}$

$I_{i} \dot{w}=\sum_{j} R_{i} \hat{n}_{i j} \times F_{i j}$

In equation (1), the left-hand side is the inertia of grain $i$. In the right hand side, the first term is the gravitational force, the second term is the buoyancy force whose magnitude depends on the immersed volume of the grain $V_{i m m}$, and on the fluid density $\rho_{f}$, the third term is the sum of contact forces with all grains $j$ that are in contact with grain $i$, and the fourth term, which was not included in Clément et al. 2017a,b, represents the drag force exerted by the fluid pressure gradient, $\nabla P$, where $P$ is the pore pressure deviation from hydrostatic values and $V_{i}$ is the volume of grain $i$.

Fluid Phase. The interstitial fluid is modeled as a continuum on a superimposed Eulerian grid (McNamara et al. 2000). The grid spacing is set to be two grain diameters, to both respect Darcy's law over each grid cell and to allow sufficient resolution for the fluid solver (Goren et al. 2011). The fluid pressure equation is (Goren et al. 2010, 2011, Niebling et al. 2010a,b):

$\frac{\partial P}{\partial t}=\frac{1}{\beta \phi \eta} \nabla \cdot[k \nabla P]-\frac{1}{\beta \phi} \nabla \cdot \bar{u}_{s}$

The left-hand side of equation (3) represents the temporal derivative of the pore pressure. On the right-hand side, the first term represents pore pressure diffusion, where $k$ is the permeability, $\beta$ is the fluid compressibility, and $\eta$ is the fluid dynamic viscosity. The second term represents a source for pore pressure due to the solid grain velocity divergence.

Coupling Between the Solid and The Fluid Phases. In order to achieve two-way coupling between the two phases, the relevant quantities are interpolated between the 
grains and the fluid grid. The porosity and the solid grain velocity are defined on the fluid grid via a bi-linear interpolation scheme that assures smooth porosity and solid velocity fields. This allows solving eq. (3) for the spatially and temporally variable PP. In addition, to solve eq. (1), the pressure gradient is interpolated back from the fluid grid to the grains together with the porosity $\phi$ using the same bi-linear scheme. In our model, the permeability and porosity are connected by the 3D Carman-Kozeny relationship:

$k=\alpha \overline{r^{2}} \frac{\phi^{3}}{(1-\phi)^{2}}$

Where $\overline{r^{2}}$ is the average of the squared grain radii in the surroundings, and $\alpha$ is a constant that allows us to vary permeability between different simulations, while keeping the characteristic time scale of pressure diffusion across grid spacing longer than model time step.

Treating the Intruder. As the intruder is larger than the grid spacing, we cannot directly use the previously described solid-fluid interpolation scheme to account for its contribution to porosity and solid velocity on grid nodes, and for the back interpolation of defining the pressure gradient over it based on the surrounding grid nodes. To overcome this limitation, we treat the intruder as a cluster of polygonal grains glued together, where each polygon corresponds to the area of intersect between the intruder and a cell (see Fig 1). To achieve this, we define virtual grains with mass, volume, and center of mass based on the volume and location of each polygon that results from intersecting the intruder with a particular cell, where virtual grains velocity is equivalent to the intruder velocity. Then, the virtual intruder equivalent grains can be treated as normal grains in the interpolation scheme of solid grains to fluid grid. For numerical stability reasons, we assign a finite porosity of $\phi=0.001$ in cells that are fully covered by the intruder. This low porosity ensures that the permeability inside the intruder as calculated in eq. 4 , will be sufficiently small relative to the permeability in the rest of the medium. The fluid pressure equation (3) can thus be solved continuously over the full domain, including the intruder, that acts as a low permeability barrier for fluid flow. The pressure gradient force on the center of mass of the intruder is calculated using the mean value of pressure gradient on grid nodes that are covered by the intruder. This arises from the discretization of the volume integral over the pressure gradient (eq. 5):

$\left(\frac{\nabla P \cdot V_{i}}{1-\phi}\right)_{\text {intruder }}=\frac{1}{1-\phi} \int \nabla P d V=\frac{1}{1-\phi} \sum_{k}^{n} \nabla P_{k} \frac{V}{n}=\frac{V}{(1-\phi)} \cdot \frac{1}{n} \sum_{k}^{n} \nabla P_{k}$

where $V$ is the volume of the intruder and $n$ is the number of grid cells that intersect with it. For other aspects of the calculation such as the solution of equations (1) and (2), the intruder is treated as a thin disc with thickness of one average grain diameter. 


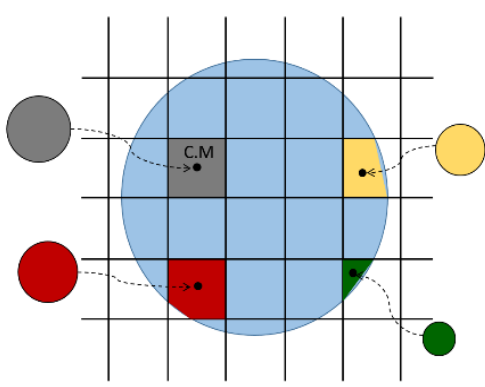

Figure 1. Partitioning the intruder into virtual grains. Each polygon that forms from the intersection between the grid and the intruder translates into a virtual grain with equivalent area, mass, center of mass (C.M) and velocity. In the next stage, these virtual grains contribute their quantities to the fluid nodes using the bilinear interpolation scheme.

Simulation Setup. The numerical system (Fig. 2) represents a thin columnar (HeleShaw) cell of densely packed spheres with an intruder on top. The cell is horizontally periodic. Dynamics is induced by cyclically shearing the bottom wall at a pre-defined amplitude and frequency. The water table level is maintained at a constant height. For the buoyancy effect the water level sets the degree of immersion of the grains and the intruder, and for the PP effect it is used as the top zero pressure boundary, which allows efficient fluid drainage across the top. The base of the cell is set as a no flow boundary. The physical parameters of the simulation are provided in table 1 .

Table 1. Physical parameters used in the simulations.

\begin{tabular}{|c|c|c|c|}
\hline Grain density & $\rho_{s}=2,640 \mathrm{~kg} \cdot \mathrm{m}^{-3}$ & Fluid compressibility & $\beta_{f}=4.5 \cdot 10^{-10} P a^{-1}$ \\
\hline Intruder density & $\rho_{\text {int }}=1,980 \mathrm{~kg} \cdot \mathrm{m}^{-3}$ & Fluid viscosity & $\eta=10^{-3} P a \cdot s$ \\
\hline Fluid density & $\rho_{f}=1,000 \mathrm{~kg} \cdot \mathrm{m}^{-3}$ & $\begin{array}{l}\text { Average grain } \\
\text { diameter }\end{array}$ & $d=0.01 \mathrm{~m}$ \\
\hline Young modulus & $E=10^{10} P a$ & Intruder diameter & $D=0.06 m$ \\
\hline Friction coefficient & $\mu=0.5$ & $\begin{array}{l}\text { Permeability (order } \\
\text { of magnitude) }\end{array}$ & $\kappa \sim 10^{-9}-10^{-10} m^{2}$ \\
\hline
\end{tabular}

\section{PRELIMINARY RESULTS FROM SIMULATIONS WITH PORE PRESSURE}

Figure 3 shows results of simulations with horizontal shaking frequency of $12 \mathrm{~Hz}$, that differ in their shaking amplitude and thus in their Peak Ground Acceleration (PGA) provided by the bottom wall. We quantify the amount of intruder sinking by measuring its normalized emerged volume, defined as the ratio between its instantaneous emerged volume $\left(V_{e m}\right)$ to its initial emerged volume $\left(V_{e m}(0)\right)$. We observe that as the PGA increases from $0.01 \mathrm{~g}$ to $0.1 \mathrm{~g}$, the emerged volume decreases and the intruder sinks further. Comparing simulations with and without the PP effect, we find that intruder sinking is enhanced in simulations that include dynamically induced PP (depicted by solid black lines) compared to simulations in which only the buoyancy effect is included (depicted by dashed light blue lines) as in Clément et al. 2017a,b work. While at the lower PGA the enhanced sinking is negligible, at higher PGA values, sinking becomes increasingly more significant. The RHS of Fig 3 shows the ratio of PP (in excess of 
hydrostatic) to effective normal stress $\left(\sigma_{e f f}=\left(\rho_{s}-\rho_{f}\right) g h\right.$, where $h$ is the depth with respect to the water table, and $\rho_{s}$ the bulk density of the grains) at three depths (locations shown in Fig 2), for simulations that include PP. As the PGA increases this ratio increases and is sustained longer at all depths, but importantly, this ratio never equals 1 . This means that although the total PP never reaches lithostatic values, still the elevated values of PP makes the granular layer more liquefied. This is testified by the enhanced intruder sinking, relative to the case of buoyancy alone (which in itself promotes liquefaction, but at higher PGA).

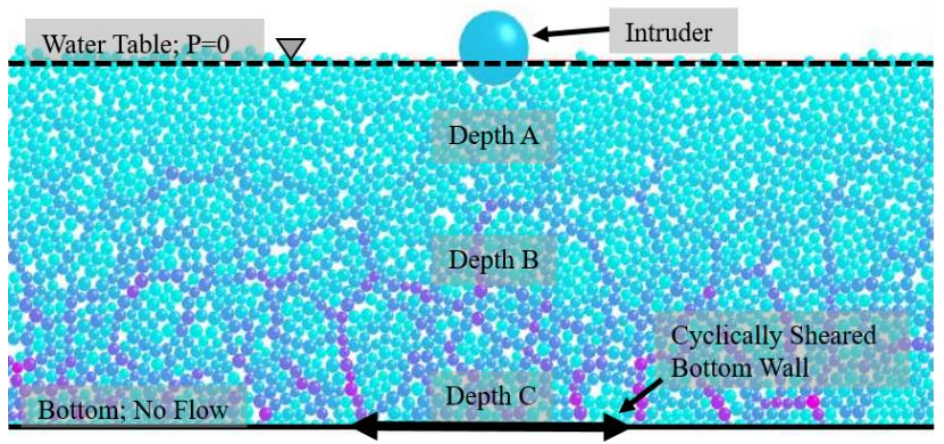

Figure 2. Numerical system setup

\section{CONCLUSIONS}

In the current work, we study mechanisms for liquefaction. For that, we adopt the field scale phenomenological definition that identifies liquefaction with observations of macro-scale changes in the rheological response of a soil, as it changes from a solid skeleton to a liquefied slurry. Here, we follow the sinking of an intruder (simulating a building) into a saturated dense and drained soil during horizontal shaking. The preliminary modeling results show that liquefaction, as testified by the intruder sinking towards its isostatic position, occurs under specific shaking conditions in saturated soils. Liquefaction does not require loose sediments, neither does it require pore pressure reaching lithostatic values. Instead, it requires the correct combination of mechanistic effects: horizontal grain accelerations, fluid buoyancy and pore-pressure rise. Clément et al. 2017a,b have shown that some sinking can occur already at relatively low PGAs, solely due to the effect of buoyancy. Yet, here we find that sinking is enhanced significantly by dynamically induced PP. This occurs even when the PP value remains below the effective normal stress, i.e., even when the classical laboratory scale liquefaction criterion of pore pressure becoming equal to the lithostatic stress, is not met. 


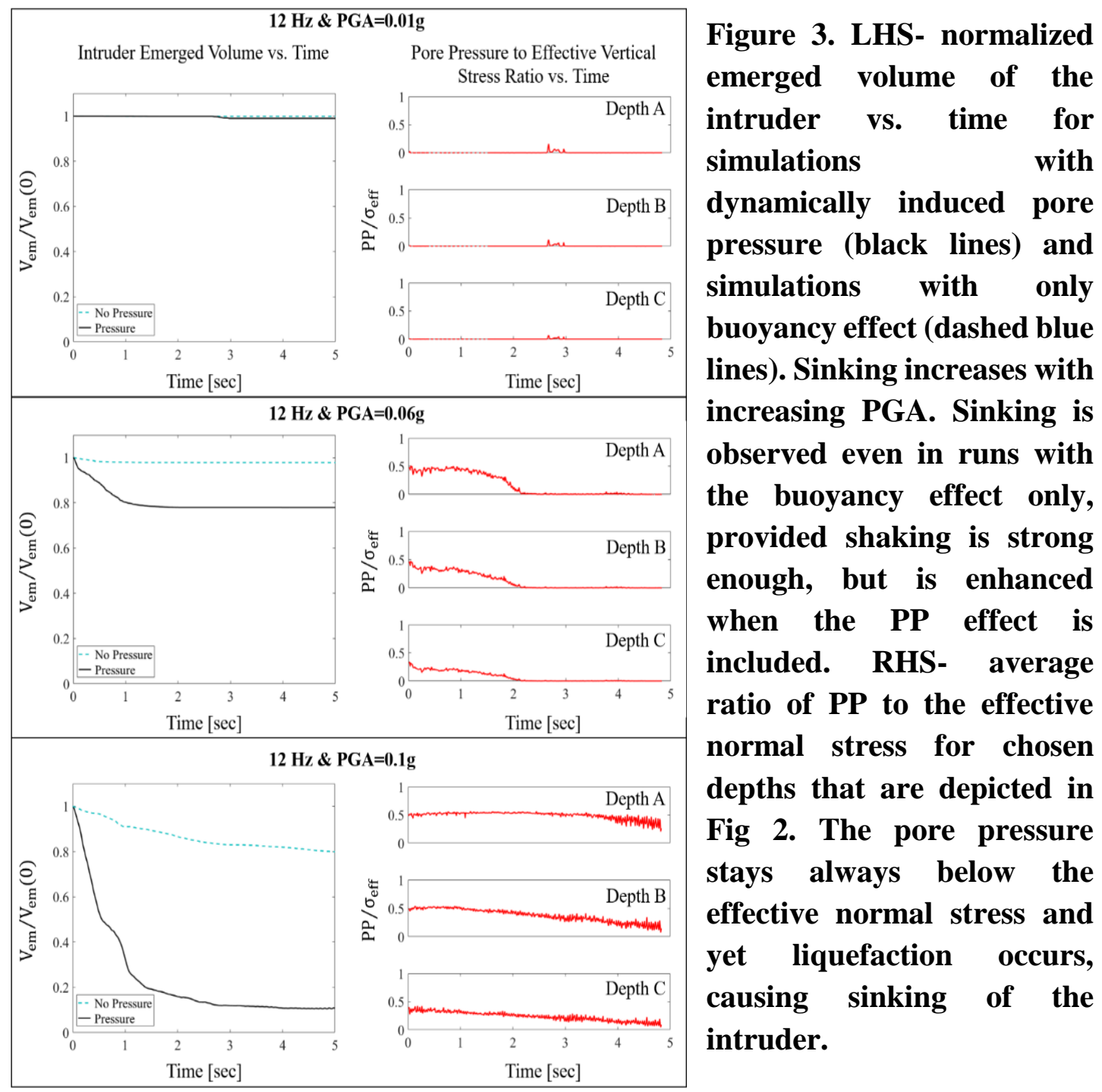

To conclude, liquefaction can occur due to high enough driving force, which overcomes the strength of the soil, or from the opposite perspective, of lowering the strength of the soil itself. The fluid effect is two-fold: The buoyancy force reduces the strength of the soil statically and uniformly, while the PP effect reduces the strength by forming dynamically.

\section{REFERENCES}

Clément, C., Toussaint, R., and Aharonov, E. (2017). "Shake and sink: liquefaction without pressurization." under-review. 
Clément, C., Toussaint, R., Stojanova, M., and Aharonov, E. (2017). "The art of sinking intruder during earthquakes." under-review.

Cundall, P.A., and Strack, O.D. (1979). "A discrete numerical model for granular assemblies." Geotechnique, 29(1), 47-65.

Goren, L., Aharonov, E., Sparks, D., and Toussaint, R. (2010). ’Pore pressure evolution in deforming granular material: A general formulation and the infinitely stiff approximation." Journal of Geophysical Research, 115(B9).

Goren, L., Aharonov, E., Sparks, D., and Toussaint, R. (2011). 'The mechanical coupling of fluid-filled granular material under shear." Pure and Applied Geophysics, 168(12), 2289-2323.

Holzer, T.L., Youd, T.L., and Hanks, T.c. (1989). 'Dynamics of liquefaction during the 1987 superstition hills, california, earthquake." Science, 244(4900), 56-59.

Martin, G.R., Finn, W.L., and Seed, H.B, (1975). " Fundementals of liquefaction under cyclic loading." Journal of Geotechnical and Geoenvironmental Engineering, 101(ASCE\# 1123 Proceedings).

McNamara, S., Flekkøy, E.G., and Måløy, K.J. (2000). ”Grains and gas flow: molecular dynamics with hydrodynamic interactions." Physical Review E, 61(4), 4054.

Niebling, M.J., Flekkøy, E.G., Måløy, K.J., and Toussaint, R. (2010). ’Mixing of a granular layer falling through a fluid." Physical Review E, 82(1), 011301.

Niebling, M.J., Flekkøy, E.G., Måløy, K.J., and Toussaint, R. (2010). "Sedimentation instabilities: Impact of the fluid compressibility and viscosity." Physical Review E, 82(1), 051302.

Obermeier, S.F. (1996). "Use of liquefaction-induced features for paleoseismic analysis - An overview of how seismic liquefaction features can be distinguished from other features and how their regional distribution and properties of source sediment can be used to infer the location and streangth of Holocene paleo-earthquakes." Engineering Geology, 44(1), 1-76.

Soga, K. (1998). "Soil liquefaction effects observed in the Kobe earthquake of 1995." Proceedings of the ICE Geotechnical Engineering, 131, 34-51.

Wang, Y.C. (2007). "Liquefaction beyond the near field." Seismological Reasearch Letters, 78, 512-517.

Youd, T.L., and Idriss, I.M. (2001). " Liquefaction Resistance of Soils: Summary Report from the 1996 NCEER and 1998 NCEER/NSF Workshops on Evaluation of Liquefaction Resistance of Soils." Journal of Geotechnical and Geoenvironmental Engineering, 127(10), 817-83 